\title{
How bursting and tonic dopaminergic activity generates LTP and
} LTD

\section{Adedoyin Thompson*1, Bernd Porr ${ }^{1}$, Alice Egerton ${ }^{2}$ and Florentin Wörgöter ${ }^{2}$}

\author{
Address: ${ }^{1}$ Department of Electronics and Electrical Engineering, University of Glasgow, Glasgow, G12 8LT, UK and ${ }^{2}$ Bernstein Center for \\ Computational Neuroscience, University of Göttingen, Göttingen, Germany \\ Email: Adedoyin Thompson* - mariat@elec.gla.ac.uk \\ * Corresponding author
}

from Sixteenth Annual Computational Neuroscience Meeting: CNS*2007

Toronto, Canada. 7-12 July 2007

Published: 6 July 2007

BMC Neuroscience 2007, 8(Suppl 2):P88 doi:10.1 I86/147|-2202-8-S2-P88

(c) 2007 Thompson et al; licensee BioMed Central Ltd.

Dopaminergic activity has commonly been interpreted as an error signal in which an increased activity codes a positive error [1] i.e. long term potentiation (LTP), and a decrease in dopamine concentration codes a negative error [1] i.e. long term depression (LTD). Recent experimental evidence in the cortical and sub-cortical areas of the brain [2], propose a different method of generating LTP or LTD by dopaminergic activity. It is suggested that LTP or LTD are controlled not by the quantity, but by the rate of the dopaminergic activity. A model of the sub-cortical nuclei of the limbic system has been shown to implement a process in which learning and reverse learning of reward stimulus associations can be achieved. The following nuclei are implemented: The nucleus accumbens (NAcc), with its sub units NAcc shell and NAcc core and the ventral tegmental area (VTA), which provides a Dopamine (DA) input to the Nacc. During learning, bursting dopaminergic activity will dominate and cause LTP, whereas during reverse learning, tonic dopaminergic activity will cause LTD. This new coding of dopaminergic activity has been implemented in the limbic system circuitry and has successfully been shown to implement a process in which learning and reverse learning of reward stimulus associations can be achieved.

\section{References}

I. Schultz W, Dayan P, Read Montague P: A neural substrate of prediction and reward. Science 1997, 275:1593-1599.

2. Cragg S: Meaningful silences: how dopamine listens to the ACh pause. Trends Neurosci 2006, 29(3): $125-131$. 